The Israeli Journal of Aquaculture - Bamidgeh, IJA_71.2020.1126881, 12 pages

CCBY-NC-ND-4.0 • https://doi.org/10.46989/001c.21471

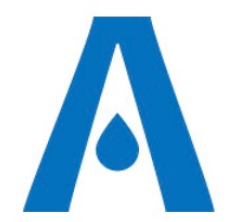

The $I J A$ is a peer-reviewed open-access, electronic journal, freely available without charge to users

Produced by the AquacultureHub non-profit Foundation Sale of IJA papers is strictly forbidden

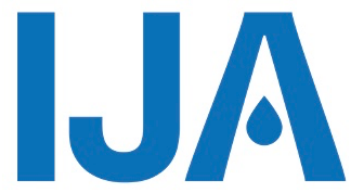

\title{
Molecular cloning and functional analysis of type I IFNd of Chinese sea bass (Lateolabrax maculatus) under immune stimulation
}

\section{Yaqing Zhang ${ }^{1,2}$, Lulu Yan², Pengfei Wang ${ }^{2}$, Chao Zhao², Sigang Fan $^{2}$, Man Yuan 1,2, GaoFeng Qiu' ${ }^{1}$, Lihua Qiu1,2,3*}

${ }^{1}$ National Demonstration Center for Experimental Fisheries Science Education, Key Laboratory of Exploration and Utilization of Aquatic Genetic Resources, Ministry of Education, Key Laboratory of Freshwater Aquatic Genetic Resources, Ministry of Agriculture, Shanghai Engineering Research Center of Aquaculture, Shanghai Ocean University, Shanghai, 201306, China

${ }^{2}$ Key Laboratory of South China Sea Fishery Resources Exploitation \& Utilization, Ministry of Agriculture and Rural Affairs, South China Sea Fisheries Research Institute, Chinese Academy of Fishery Sciences, Guangzhou, 510300, PR China

${ }^{3}$ Guangdong Provincial Key Laboratory of Fishery Ecology and Environment, Guangzhou, 510000, P.R. China

Key words: Lateolabrax maculatus; Interferon; Complete cDNA; Innate immunity

\begin{abstract}
Chinese sea bass (Lateolabrax maculatus) is an economically important marine cultured species in China. Interferons (IFNs) play an essential role in innate antiviral immunity. The study on IFN immune system helps prevent and control viral diseases of $L$. maculatus. We have obtained cloning and characterization of the type I IFNd gene from L. maculatus ( $L m I F N d$ ) in the present study. The full length of cDNA was $1190 \mathrm{bp}$, including 5'UTR (untranslated region) of 354 bp, 3'UTR of $278 \mathrm{bp}$, and an open reading frame (ORF) of $558 \mathrm{bp}$. It encodes 185 amino acids, and the first 20 amino acids are hypothetical signal peptides. The results of amino acid multiple sequence alignment and phylogenetic tree analysis showed that LmIFNd and mandarin fish (Siniperca chuatsi) IFNd were clustered into one branch, and the gene sequence similarity was as high as $88.9 \%$. The expression of LmIFNd was tissue-specific and highly expressed in the head kidney, spleen, and gill. After infection with Rana grylio virus (RGV), and polyinosinic-polycytidylic acid [Poly(I:C)], the expression of LmIFNd in gill, spleen, and head-kidney was up-regulated significantly. Besides, the expression level of LmIFNd has increased significantly under the stimulation of Vibrio harveyi and Streptococcus iniae. The results show that LmIFNd may play a protective role in both viral and bacterial infections.
\end{abstract}

* Corresponding author. LiHua Qiu, e-mail: qiugroup_bio@outlook.com 


\section{Introduction}

Lateolabrax maculatus is one of the most economically important farmed fish in China. It exhibits a fast growth rate, short culture cycle, delicious meat and high economic benefits. According to the 2019 China Fisheries Statistical Yearbook, the total output of L. maculatus culture in China exceeded 160,000 tons in 2018, ranking second among mariculture economic fish, with highest production in Guangdong, Fujian, Zhejiang, Guangxi and Shandong. Most L. maculatus culture methods involve high-density intensive culture. However, this scale of aquaculture makes fish especially vulnerable to viral or bacterial infection, resulting in high mortality and huge economic losses to the aquaculture industry. The innate immune system is the first line of defense for resisting invading microorganisms in vertebrates. In addition, IFNs are among the most critical innate immune cytokines. Therefore, studying the IFN immune system of $L$. maculatus helps to prevent and control viral diseases of L. maculatus.

Interferons (IFNs) are a type II a-helical cytokine with multiple biological effects. IFNs play a variety of roles in numerous cellular responses by inducing a large number of IFN stimulating factors, including innate immune cell activation and regulation of adaptive immune response, especially antiviral responses (Luo et al., 2018; Teijaro, 2016). When the body is infected by a virus, the cells secrete the bioactive cytokine IFN, to induce innate immunity (Samuel, 2001). According to sequence similarity, genomic organization, receptor usage and biological function, mammalian IFNs can be divided into type I, type II and type III (Pestka S, 2004). Functionally, types I and III IFNs are specialized as innate antiviral factors (Teijaro, 2016), while type II IFNs are considered to be cytokines that regulate innate and adaptive immunity against viral and intracellular bacterial infections (Schoenborn et al., 2007). IFNs have been found in vertebrates ranging from fish to mammals. Type II IFNs are uniquely composed of IFN- $\gamma$ in mammals, but not in fish. It has been reported that there are two IFN components in fish: IFN- $\gamma$ and IFN- $\gamma$-related genes ( $\mathrm{Li}$ et al., 2019; Zou et al., 2011). In mammals, IFN-Y acts as a key immunomodulatory factor, participating in the immune response to a variety of pathogens (viruses, bacteria and protozoa), as well as cell growth, cell death and anti-tumor activity (Bae et al., 2016; Li et al., 2019). However, type III IFNs are limited to tissues with a high risk of virus exposure and infection, such as tissues with an epithelial surface, but type III IFN genes have not been found in teleost fish (Secombes et al., 2017). According to the relationship between the amount of cysteine and phylogeny, it is generally believed that type I IFNs are divided into group I and group II. There are two cysteines in group I and four cysteines in group II, and these can be further divided into subgroups a, d, e and $h$ and b, $c$ and f, respectively (Laghari et al., 2018; Milne et al., 2018). The type I IFNs of most fishes consist of three subgroups $\mathrm{a}, \mathrm{c}$ and $\mathrm{d}$, and the recently discovered IFN $h$ is only found in Perciformes (Milne et al., 2018). Type I IFNs are a secreted cytokine that coordinates various immune responses to infection. Although type I IFNs are generally considered to be the most important factor in viral response, most bacterial pathogens can also induce type I IFNs, and some genes induced by type I IFNs also display antibacterial activity, such as p47GTPases (Taylor et al., 2004). The signal pathways of three kinds of IFNs are mediated by three different receptor complexes. Type I IFNs bind to the same protein complex IFN-a receptor (IFNAR) consisting of two subunits of the receptor chains IFNAR1 and IFNAR2. A tetramer consisting of two IFN- $y$ receptor 2 (IFNGR2) chains and two IFNGR1 chains binds dimers of type II IFN-y. The interleukin-10 receptor 2 (IL-10R2) associates with IFN- $\lambda$ receptor 1 (IFNLR1) to bind the type III IFN- $\lambda$ (Sadler et al., 2008). From an evolutionary point of view, mammalian type I IFNs play a role mainly through the JAK-STAT pathway (Stark GR, 2012). Some studies have shown that tyrosine kinase 2 (TYK2), Janus kinase 1 (JAK1), signal transducers and activators of transcription (STAT1, STAT2) and IFN regulator factor (IRF) 9 which are involved in the type I IFN-mediated signal transduction pathway also exist in fish (Jin et al., 2018), and this mechanism can be preserved in fish (Hou et al., 2017). 
The first attempts at fish type I IFN gene cloning were made in the early 1990s, but were unsuccessful. It was not until 2003 that three different research teams successfully cloned the fish type I IFN gene from Atlantic salmon (Salmo solar) (Stein et al., 2007), zebrafish (Danio rerio) (Altmann SM, 2003) and spotted green puffer fish (Tetraodon nigroviridis) (Lutfalla et al., 2003). Subsequently, the IFN genes of grass carp (Ctenopharyngodon idella), common carp (Cyprinus carpio), silver crucian carp (Carassiusaur atus gibelio), red crucian carp (Carassius auratus gibelio) were identified (Casani et al., 2009; Chang et al., 2009; Long et al., 2006; Purcell et al., 2009). In recent years, fish type I IFN has been a focus of research for comparative immunologists, and great progress has been made in the study of type I interferon system. For example, Ctenopharyngodon idella recombinant interferon protein (rCiIFN) can induce the expression of some well-known ISGs and IFNs in vivo and in vitro. In addition, both intraperitoneal injection and oral administration of rCiIFN can effectively improve the survival rate of fish infected with GCHV (Dongming Li et al., 2013). In mandarin fish (Siniperca chuatsi), the expression of three IFNs (IFNc, IFNd and IFNh) induced by intraperitoneal injection of Poly(I:C), which mimics dsRNA virus infection, was significantly induced in the head kidney (Laghari et al., 2018).

The present study provides the first report of the CDNA sequence of LmIFNd and the characteristics of LmIFNd expression in different tissues and pathogeny microbiology, which provides a molecular theoretical basis for research on the immunity of $L$. maculatus.

\section{Materials and Methods}

Ethics statement. L. maculatus is not an endangered or protected species, and there is no requirement for permission to undertake experiments involving this species in China.

Experimental animal. The $L$. maculatus for the experiment were provided by the Zhuhai Experimental Base of the South China Sea Fisheries Research Institute, China Academy of Fisheries Sciences (Guangdong, China). The fish were $35 \pm 5 \mathrm{~cm}$ long and weighed $365 \pm$ $10 \mathrm{~g}$. Groups of 15 fish were reared in yellow round vats with a water temperature of 24 $\pm 1{ }^{\circ} \mathrm{C}$ and a salinity of $2.88 \%$. The seawater was continuously aerated and was changed every day. L. maculatus were acclimatized for one week prior to the experiment.

Virus preparation. Diseased tissue, $0.4 \mathrm{~g}$ Rana grylio virus (RGV), and $4 \mathrm{ml}$ aseptic PBS were placed in a sterile and enzyme-free glass grinding tube, ground slowly on ice, frozen and thawed twice at $-20{ }^{\circ} \mathrm{C}$. The homogenate was then transferred into a centrifuge tube at $4{ }^{\circ} \mathrm{C}$, and centrifuged at $3000 \mathrm{r} / \mathrm{min}$ for $20 \mathrm{~min}$. The supernatant was removed and centrifuged at $8000 \mathrm{r} / \mathrm{min}$ for $25 \mathrm{~min}$. The supernatant was filtered with $0.45 \mu \mathrm{m}$ and 0.22 $\mu \mathrm{m}$ filters, respectively, diluted 10 times with aseptic PBS and prepared at $-20{ }^{\circ} \mathrm{C}$.

Bacteria preparation. Vibrio harveyi and Streptococcus iniae were provided by the Department of fishery biological disease prevention and control, South China Sea Fisheries Research Institute, China Academy of Fisheries Sciences. During the experiment, the two strains were activated by marking on the plate, and single colonies of $V$. harvei and $S$. iniae were inoculated into liquid $\mathrm{LB}$ and $\mathrm{BHI}$ mediums, respectively, at $28{ }^{\circ} \mathrm{C}, 180 \mathrm{rpm}$, and 0.6 OD600. The bacteria were collected and washed twice with aseptic PBS. Then the bacterial body weight was prepared into a bacterial suspension and placed at $20^{\circ} \mathrm{C}$ until required.

Design of injection experiment. To detect the mRNA expression pattern of LmIFNd after viral and bacterial infection, the fish were divided into five groups. Group 1 (PBS group) comprised 30 fish that received an intraperitoneal injection with $800 \mu \mathrm{L}$ sterile PBS (control). Group 2 (RGV group) contained 30 fish that received an intraperitoneal injection with $800 \mu \mathrm{L}$ RGV virus $\left(3.0 \times 10^{8}\right.$ copies $/ \mu \mathrm{L}$ ). Group 3 (Poly(I:C) group) comprised 30 fish that received an intraperitoneal injection with $800 \mu \mathrm{L}$ Poly(I:C) $(1 \mathrm{mg} / \mathrm{mL})$. Group $4(V$. harvei group) comprised 30 fish that received an intraperitoneal injection with $800 \mu \mathrm{L} V$. harvei $\left(5 \times 10^{9} \mathrm{cfu} / \mathrm{mL}\right)$. Group 5 (S. iniae group) contained 30 fish that received an intraperitoneal injection with $800 \mu \mathrm{L} \mathrm{S}$. iniae $\left(5 \times 10^{9} \mathrm{cfu} / \mathrm{mL}\right)$.

sample collection. To collect tissue samples from healthy L. maculatus, three healthy L. maculatus were randomly selected and anesthetized with $50 \mathrm{mg} / \mathrm{ml} \mathrm{MS} 222$ for $2 \mathrm{~min}$. Blood, spleen, head-kidney, liver, gill, heart, muscle, small intestine, skin, stomach and 
other tissues were removed and immediately put into liquid nitrogen for temporary storage, and then transferred to a $-80^{\circ} \mathrm{C}$ refrigerator until further analysis.

Tissue samples were collected from three $L$. maculatus from the PBS group, RGV group, Poly(I:C) group, $V$. harvei group and $S$. iniae group. Gill, head kidney and spleen tissues were removed and immediately put into liquid nitrogen for temporary storage at $0 \mathrm{~h}$ (the immediately after infection), $6 \mathrm{~h}, 12 \mathrm{~h}, 24 \mathrm{~h}, 48 \mathrm{~h}, 72 \mathrm{~h}$, and $96 \mathrm{~h}$.

Total RNA extraction and CDNA Synthesis.

Approximately $50 \mathrm{mg}$ of total RNA was extracted from each tissue sample using Trizol reagent (Invitrogen, USA) following the manufacturer's instructions. The integrity of RNA was detected by $1.2 \%$ agarose gel electrophoresis, and total RNA concentration was estimated at $260 \mathrm{~nm}$ using a NanoDrop-2000 (Thermo Fisher, USA). Reverse transcription with $1 \mu \mathrm{g}$ of total RNA was performed using TaKaRa kit (Primer Script RT Reagent kit with gDNA Eraser, TaKaRa, Japan) following the manufacturer's instructions. The synthesized CDNA was stored at $-20^{\circ} \mathrm{C}$.

The full-length cDNA cloning of LmIFNd. The partial CDNA sequence of type I IFNd was obtained from the transcriptome database. The open reading frame (ORF) sequence was amplified using IFN-OF/OR primers (Table 1), which was synthesized by Sangon Biotech. The cDNA template for rapid amplification of the CDNA ends (RACE) was synthesized according to the instructions of the SMARTTM RACE $5^{\prime} / 3^{\prime}$ kit (Clontech, Japan). This step reaction was performed by touchdown PCR and nested PCR using the 5'IFNR1/ 5'IFNR2 /3'IFNF1 primers and UPM/NUP (Table 1). The RACE - PCR products were purified using an agarose gel DNA purification kit (Sangon Biotech, China). The product was ligated to PMD18-T vector and transformed into Escherichia coli DH5 a competent cells. The positive colonies were selected and sent to the Guangzhou Ruibo Biological Company for sequencing.

The 5 'and 3' end sequences and the ORF of LmIFNd were spliced to get the full length of LmIFNd. Using the specific primers CF1 and CR1, the 5'RACE SMARTTM CDNA (diluted 10 times), was used as the template to verify the correctness of the spliced IFN full-length cDNA sequence.

Bioinformatics analysis of LmIFNd. The LmIFNd CDNA sequences were compared and analyzed using an online BLAST analysis tool https:/blast.ncbi.nlm.nih.gov/ Blast.cgi. The ORF and the coding amino acid sequence was predicted by NCBI online ORF Finder https://www.ncbi.nlm.nih.gov/orffinder/. The ExPaSy calculation tool was used to calculate the theoretical isoelectric point and molecular weight http://web.expasy.org/compute pi/. The SignalIP4.1 tool was used to predict the signal peptide http://www.cbs.dtu.dk/services/SignalP/. And SMART http://smart.embl-heidelberg.de/ was used to predict the LmIFNd domain. Multiple sequence alignment of LmIFNd was performed using Clustal Omega and Jalview software, and the phylogenetic tree was constructed using neighbor joining by Mega7.0. All of the IFN alignment sequence information was downloaded from the GenBank database. The GenBank accession numbers of the IFNs from different species are as follows: O. mykiss IFNa3 (ACJ03567), S. salar IFNa1 (NP_001117182), S. salar IFNa2 (NP_001117042), S. salar IFNa3 (ACE75687), 0. mykiss IFNb3 (NP_001153974.1), O. mykiss IFNb2 (NP_001158515), O. mykiss IFNb3 (CCV17399), S. salar IFNb1 (ACE75691.1), S. salar IFNb2 (ACE75693), S. salar IFNb3 (ACE75689), O. mykiss IFN f1 (CCV17413), O. mykiss IFN f2 (CCV17414), Paralichthys olivaceus IFN3 (BBA46271), Siniperca chuatsi IFNc (AVJ47959), O. mykiss IFNc1 (CCV17402), O. mykiss IFNc2 (CCV17403.1), S. salar IFNc1 (ACE75692.1), S. salar IFNc2 (ACE75694.1), S. salar IFNc3 (ACE75688), O. mykiss IFNd (NP_001152811.1), O. mykiss IFNe1 (CCV17406), O. mykiss IFNe2 (CCV17407), O. mykiss IFNe3 (CCV17408), O. mykiss IFNe4 (CCV17409.1), O. mykiss IFNe5 (CCV17410), Larimichthys crocea IFNh (API68650), S. chuatsi type IFNh (AVJ47961), S. chuatsi IFNd (KY768915), C. carpio (BAG68522), Hyporthodus septemfasciatus (BAJ79339), T. nigroviridis IFN (CAD67779), Ictalurus punctatus IFN (AAP92146), C. idella IFN (ABC87312), Carassius auratus IFN 
(AAR20886.1), Epinephelus coioides IFN2 (KC495073), E. coioides IFN1 (KC495072), L. crocea IFNd (API68651), Lateolabrax maculatus IFNd (MT462156), Argyrosomus regius IFNd (MG489873), P. olivaceus IFN1 (BAH84776.1), P. olivaceus IFN2 (AHB59752.1), P. olivaceus IFN4 (BBA46272.1), Nothobranchius guentheri IFN1 (KP324755), Mus musculus IFN-beta (AAA37891.1), Homo sapiens interferon beta (NP_002167.1).

Spatial and temporal expression analysis of LmIFNd. The distribution and expression of LmIFNd in healthy L. maculatus and the LmIFNd mRNA expression patterns in different tissues at different time points after viral and bacterial infection were analyzed by realtime PCR (qRT-PCR) using qF1/qR1 primers (Table 1). 18sRNA was the internal reference gene, qRT-PCR amplifications were carried out on a 384-well rotor with a total volume of $12.5 \mu \mathrm{L}$, containing 6.25 $\mu \mathrm{L}$ of TB Green Premix Ex Taq II (TaKaRa) (2X), $0.5 \mu \mathrm{L}$ of each specific primer, $2 \mu \mathrm{L}$ cDNA of template, and $3.25 \mu \mathrm{L}$ of DEPC water. The PCR reaction conditions were $95^{\circ} \mathrm{C}$ for $30 \mathrm{~s}$, followed by 40 cycles of $94^{\circ} \mathrm{C}$ for $5 \mathrm{~s}, 60^{\circ} \mathrm{C}$ for $30 \mathrm{~s}$. At the end of the reaction, melting-curve analysis for each amplification was performed from 75$95^{\circ} \mathrm{C}$ to ensure specificity. Each experiment was repeated three times and three parallel reactions were carried out each time.

Statistical analyses. The relative mRNA expression data were analyzed by the $2^{-\Delta \Delta C t}$ method after normalization of $18 \mathrm{~S}$ RNA. One-way analysis of variance (ANOVA) was performed to calculate $P$ values using SPSS v22.0 software. The results of the analyses were plotted with GraphPad prism8 software.

\section{Results}

Molecular characteristics of the full-length LmIFNd gene. The full-length cDNA of the LmIFNd (GenBank No. MT462156) is 1190 bp, which contains 5'UTR (untranslated region) of $354 \mathrm{bp}, 3$ 'UTR of $278 \mathrm{bp}$ and an ORF of $558 \mathrm{bp}$. It encodes 185 amino acids. The predicted molecular weight of the LmIFNd protein is $20.977 \mathrm{kDa}$ and its theoretical isoelectric point is 6.35. There are nine unstable ATTTA mRNA sequences and one tailed signal AATAAA in the 3'UTR. The first 20 amino acids (MLNRIFFVCLFLGLYSAASS) of the Nterminal of the LmIFNd protein was predicted as a signal peptide by SignalIP. The functional domain predicts that the protein has an extracellular IFabd domain, which is a cytokine receptor binding site. Online prediction of glycosylation sites showed that there were two potential N-glycosylation sites (Asn47 and Asn50). In addition, LmIFNd has two conserved cysteine residues (Figure 1A).

Multiple sequence alignment and evolutionary tree analysis. The alignment of the deduced amino acid sequences of LmIFNd with known IFNs from other species is shown in Figure 1B. LmIFNd has the highest genetic similarity with $A$. regius IFNd $(89.9 \%)$. The similarity with S. chuatsi, D. labrax, L. calcarifer and L. crocea was $88.9 \%, 88.8 \%, 88.3 \%$ and $88.2 \%$, respectively. It was more than $80 \%$ consistent with $S$. aurata, $P$. olivaceus and $E$. septemfasciatus, indicating that the IFN gene is conservative in fish. Amino acid alignment analysis revealed that the amino acid sequence of LmIFNd is similar to that of type I IFN of other known species, with two cysteine residues.

The results of the LmIFNd phylogenetic tree showed (Figure 1C) that fish and mammals are divided into two branches. The members of the first group of fish type I IFN a, d, e and h were clustered into a small branch, and LmIFNd was closest to S. chautsi IFNd, followed by $L$. crocea and $A$. regius IFNd, and distant from $O$. mykiss IFNd. The IFN genes of fish and mammals come from the same group but have evolved differently.

Tissue distribution of LmIFNd. The spatial expression pattern of LmIFNd mRNA was determined by qRT-PCR using 18s RNA as an internal control. The brain was set to the calibrator and the variation in expression level of LmIFNd from the brain was defined as 1.00. The highest relative expression was in the head-kidney, followed by spleen and gill, 
a relatively low expression pattern was detected the brain, skin, intestine, and heart. (Figure 2).

Induced expression of LmIFNd by virus challenge. To investigate the correlation with virus infection, the expression of LmIFNd was investigated in vivo during RGV infection and poly(I:C) stimulation. Under the stimulation of RGV and Poly(I:C), the relative expression of LmIFNd in the gill, head-kidney and spleen was significantly upregulated compared with the control group, but the expression pattern was different. In the gill, the relative expression of LmIFNd increased significantly at $6 \mathrm{~h}$, peaked at $12 \mathrm{~h}$ after RGV stimulation, then decreased significantly after $24 \mathrm{~h}$. However, in the Poly(I:C) group, the relative expression level of LmIFNd began to increase significantly at $6 \mathrm{~h}$, and reached its peak at $48 \mathrm{~h}$ (Figure 3A). In the head-kidney, the relative expression of LmIFNd peaked at $6 \mathrm{~h}$ and remained significantly higher than the PBS group from 12-24 h (Figure 3B). In the spleen, the relative expression levels of LmIFNd began to increase significantly at $6 \mathrm{~h}$. However, levels began to fluctuate after $6 \mathrm{~h}$ in the RGV group, and were significantly higher than those in the control group at $96 \mathrm{~h}$. In the Poly (I:C) group, the expression of LmIFNd decreased to the level of the PBS group after $6 \mathrm{~h}$ (Figure 3C).

Induced expression of LmIFNd by bacteria challenge. To explore whether LmIFNd is involved in antibacterial activity, the expression of LmIFNd in vivo after $V$. harvei and $S$. iniae stimulation was explored. Under the stimulation of $V$. harvei and $S$. iniae, the temporal and spatial expression patterns of LmIFNd were significantly different in the gill, head-kidney and spleen. In the gill, the relative expression of LmIFNd in the S. iniae group began to increase, and peaked at $6 \mathrm{~h}$ before gradually decreasing. The relative expression of $L$ IFIFNd began to increase at $6 \mathrm{~h}$ and changed significantly at $12 \mathrm{~h}$ in the $V$. harvei group (Figure. 4A). In the head-kidney, the relative expression of LmIFNd in the S. iniae group increased significantly after $6 \mathrm{~h}$, decreased at $12 \mathrm{~h}$ and $24 \mathrm{~h}$, and then increased significantly at $48 \mathrm{~h}$. Similarly, the expression of LmIFNd in the $V$. harvei group also increased significantly at $6 \mathrm{~h}$ and peaked at $48 \mathrm{~h}$ (Figure 4B). In the spleen, the expression levels of the $V$. harvei group and the $S$. iniae group increased significantly at $6 \mathrm{~h}$. After that, the relative expression of the $V$. harvei group decreased to initial levels, while levels in the $\mathrm{S}$. iniae group remained significant at $12 \mathrm{~h}, 24 \mathrm{~h}$ and $48 \mathrm{~h}$ (Figure 4C).

Table 1 Primer sequences.

\begin{tabular}{clc}
\hline Primer name & Primer sequence 5'-3' & Primer use \\
\hline OF & ATGCTCAACAGGATCTTCTTTGTCT & ORF Verification \\
OR & TGACAGATTTAGTTGGTGGTGAGTA 1 & ORF verification \\
5'R1 & GACCTGTTTCTCGGGCTGTA & 3'RACE \\
5'R2 & GCAGCCACCTCCTCCAGAACCTGA & 5'RACE \\
UPM-long & CTAATACGACTCACTATAGGGC- & 5'RACE \\
UPM-short & CAAATACAGTGGTATCAACGCAGAGT & 3' \& 5' RACE \\
NUP & AAGCAGTGGTATCAACGCAGAGT & 3' \& 5' RACE \\
CF1 & CGCTGCTACAGTATAAATGAGCG & 3' \&5' RACE \\
CR1 & GACTGCATGTAAGCAAATAAATGAACACA & IFN Full-length verification \\
qF1 & CTTCGCCCCTGTATTGGGAG & Real-time PCR \\
qR1 & CCATCTGGTCTGCTCTCATCA & Real-time PCR \\
\hline
\end{tabular}




\section{A}

1 ACATGGGaTGTGTTGTTGCTGTCTGCAGCAGGTTTGCAGGTTTGCAGACCTGAGAGGACT 60 61 TCATGTCCCCTTCCTCTTCCTGTTAGGCGGACAACAAAGCTCAGGCTGGTTTCACACCTC 120 121 ACAGCTTGTTAACTGTGTAGTGACTCGCACACTAAAGCGGCCACATGGAGGTTTAATGAG 180 181 CTTCGTAGTGACTTTAGAGCCGCGGGAAAGCCGCAGCCATGTGTTTCCCAGGTATGTGGA 240 241 AAATGAAAGAGGCGCGCTGCTACAGTATAAATGAGCGGCTGCAGGTCTGCTCCAACAAAC 300 301 ACCTGAAGACATCCCAATCTGCTCCAAGCAAAAGCGACTTGTACCTTTTTGAAA tg tc 360 1 M L 2

361 aacaggatcttctttgtctgcctgtttctcgggctgtacagtgcagcctcctcgctgage 420 $\begin{array}{llllllllllllllllllllll}3 & \mathbf{N} & \text { R } & \text { I } & \text { F } & \text { F } & \text { V } & \text { C } & \text { L } & \text { F } & \text { L } & \text { G } & \text { L } & \text { Y } & \text { S } & \text { A } & \text { A } & \text { S } & \text { S } & \text { L } & \text { S } & 22\end{array}$ 421 tgcagatgratgratcataagttcrgacagcacagtgaaactctttgratctgatacag 480

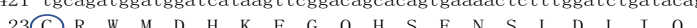
481 aggatgtctaacaactccaccaacaccactgaggatgctgaagtgaaggacactgtggec 540 $\begin{array}{llllllllllllllllllllll}43 & R & M & S & N & N & S & T & N & T & T & E & D & A & E & V & K & D & T & V & A & 62\end{array}$ 541 ttccetcataatctgtacagccaggcgtccaaagcatcagctaaggataaacttgctttc 600 $\begin{array}{llllllllllllllllllllll}63 & \mathrm{~F} & \mathrm{P} & \mathrm{H} & \mathrm{N} & \text { L } & \text { Y } & \text { S } & \text { Q } & \text { A } & \text { S } & \text { K } & \text { A } & \text { S } & \text { A } & \text { K } & \text { D } & \text { K } & \text { L } & \text { A } & \text { F } & 82\end{array}$ 601 acagttcaggttctggaggaggtggetgetctgtttgaggaggatcatagctctgetgca 660 $\begin{array}{llllllllllllllllllllll}83 & \text { T } & \text { V } & \text { Q } & \text { V } & \text { L } & \text { E } & \text { E } & \text { V } & \text { A } & \text { A } & \text { L } & \text { F } & \text { E } & \text { E } & \text { D } & \text { H } & \text { S } & \text { S } & \text { A } & \text { A } & 102\end{array}$ 661 tcatgggaggagagcacagtggaggactttgtcaatgttgtaacccagcaggctgacggc 720

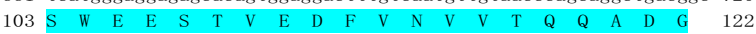
721 cttcgcccetgtattgggagccacggccacaagaagaacacaaagctgcacatgtatttc 780 $\begin{array}{lllllllllllllllllllll}123 & \text { L } & \text { R } & \text { P (C) } & \text { I } & \text { G } & \text { S } & \text { H } & \text { G } & \text { H } & \text { K } & \text { K } & \text { N } & \text { T } & \text { K } & \text { L } & \text { H } & \text { M } & \text { Y } & \text { F } & 142\end{array}$ 781 aagagactgtcgegecacgtcctaaagaaaatgggccacagtgctgaagcctgggagctg 840 $\begin{array}{llllllllllllllllllllll}143 & \text { K } & \text { R } & \text { L } & \text { S } & \text { R } & \text { H } & \text { V } & \text { L } & \text { K } & \text { K } & \text { M } & \text { G } & \text { H } & \text { S } & \text { A } & \text { E } & \text { A } & \text { W } & \text { E } & \text { L } & 162\end{array}$ 841 atcaggaaggaaatagaaactcatctgatgagagcagaccagatggtttcatctctactc 900 $\begin{array}{llllllllllllllllllllll}163 & \text { I } & R & K & \text { E } & \text { I } & \text { E } & \text { T } & \text { H } & \text { L } & \text { M } & \text { R } & \text { A } & \text { D } & \text { Q } & \text { M } & \text { V } & \text { S } & \text { S } & \text { L } & \text { L } & 182\end{array}$ 901 accaccaadtaaAATCTGTCACACTTAGAATGTGTTCATTTATTTGCTTACATGCAGTCT 960 $183 \mathrm{~T} \quad \mathrm{~T} \quad \mathrm{~N} *$ 961 ATTTATGTATTTATCTATTTATAATCAACTHATTTATTGATTTA TATCAACTTATT 1020 021 ATTTATTTCTATTGT

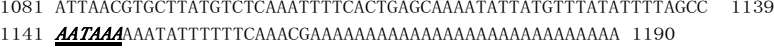

\section{C}

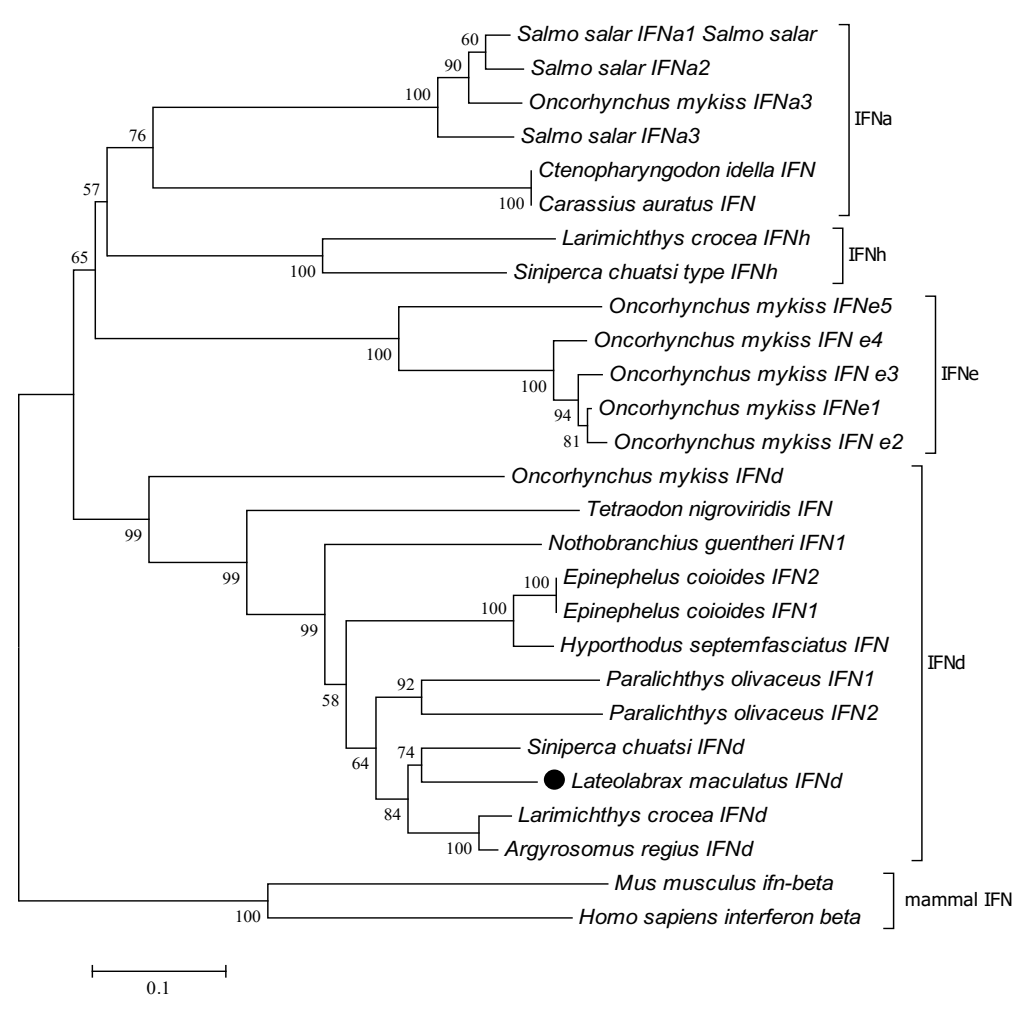

B

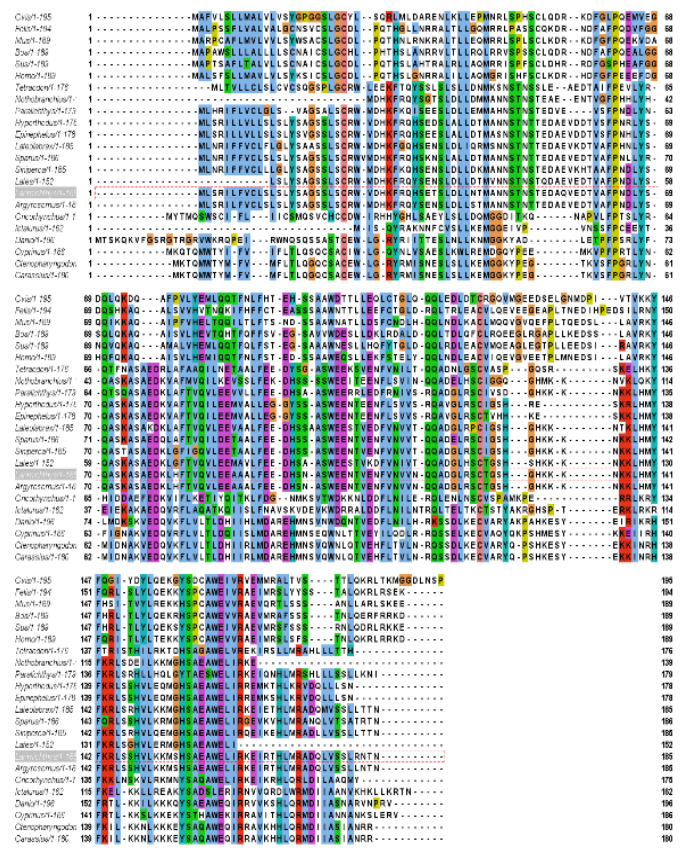

Figure 1 Molecular characterization of LmIFNd cDNA. (A)The start codon(ATG) and the stop codon(TAA) are circled in a red box, the bold font indicates the predicted signal peptide, the amino acid drawing the wavy line is the predicted $\mathrm{N}$ glycosylation site, the circle is cysteine, the blue background is the IFabd domain, the single underline is the unstable sequence, and the double underline indicates the trailing signal.(B) Multiple alignment of the amino acid sequence of LmIFNd and IFNs in the other species. (C) Phylogenetic tree of IFN in Fish and mammals. 


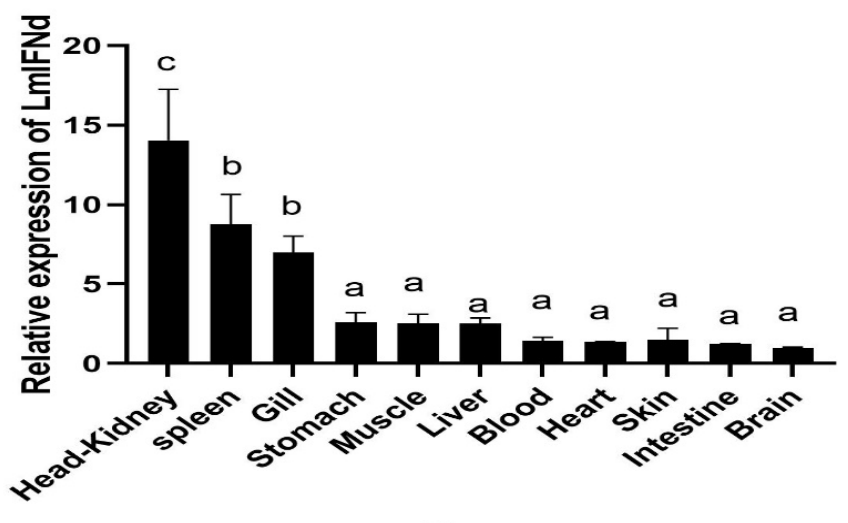

Tissue

Figure 2 Relative expression of levels of LmIFNd mRNA in different tissues

The relative expression levels in the different tissues were calculated by the $2-\Delta \Delta C t$ method and expressed as the mean \pm standard deviation $(n=3)$. Significant different letters above the vertical bars indicate difference $(P<0.05)$
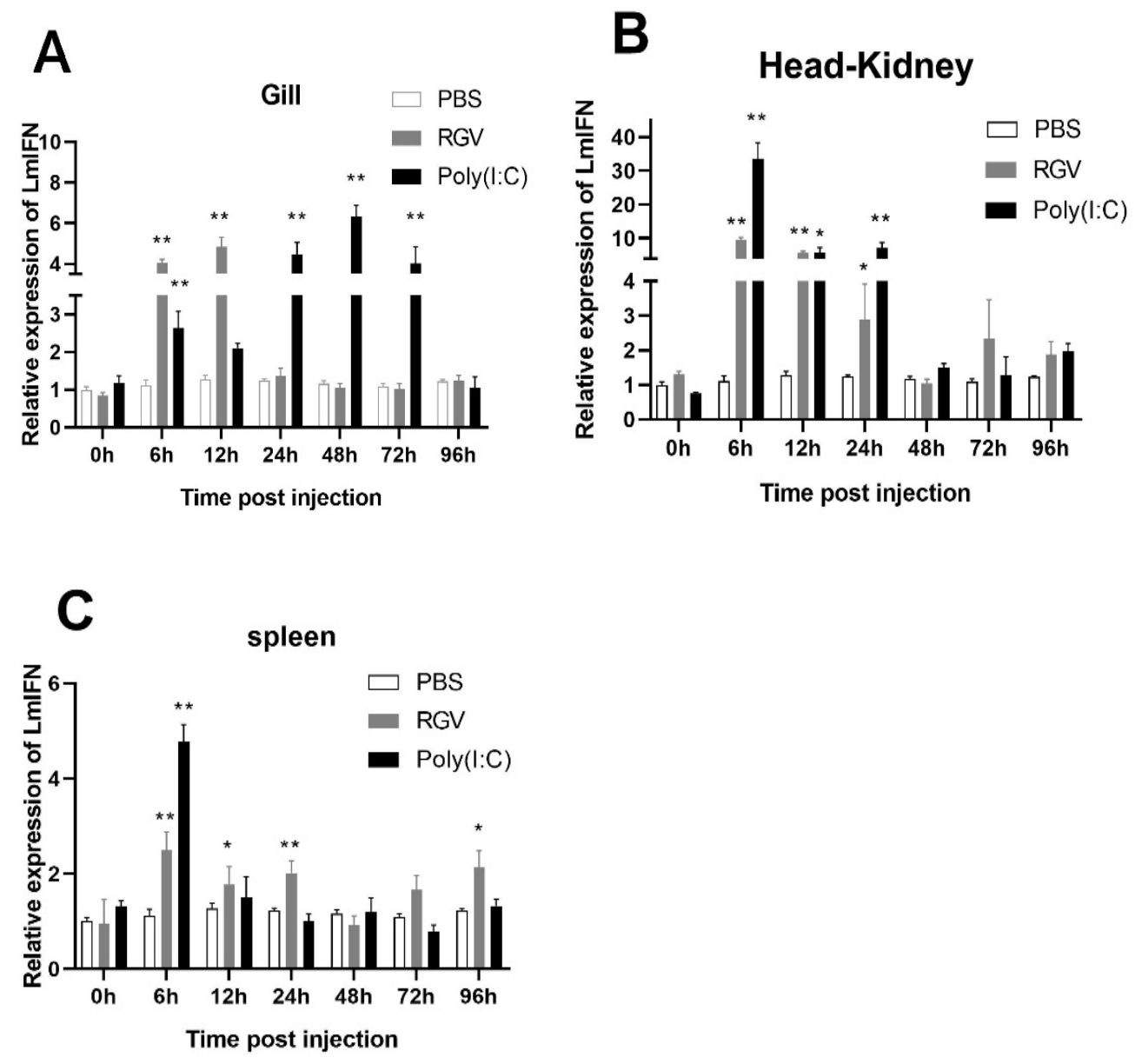

Figure 3 Spatiotemporal expression of LmIFNd mRNA in gill(A), head kidney(B) and spleen(C) of $L$. maculatus stimulated by RGV and Poly $(\mathrm{I}: C)$. The bars represent the mean \pm SD $(n=3)$. * represents significant difference $(P<0.05)$, and $* *$ represents highly significant difference $(P<0.01)$. 

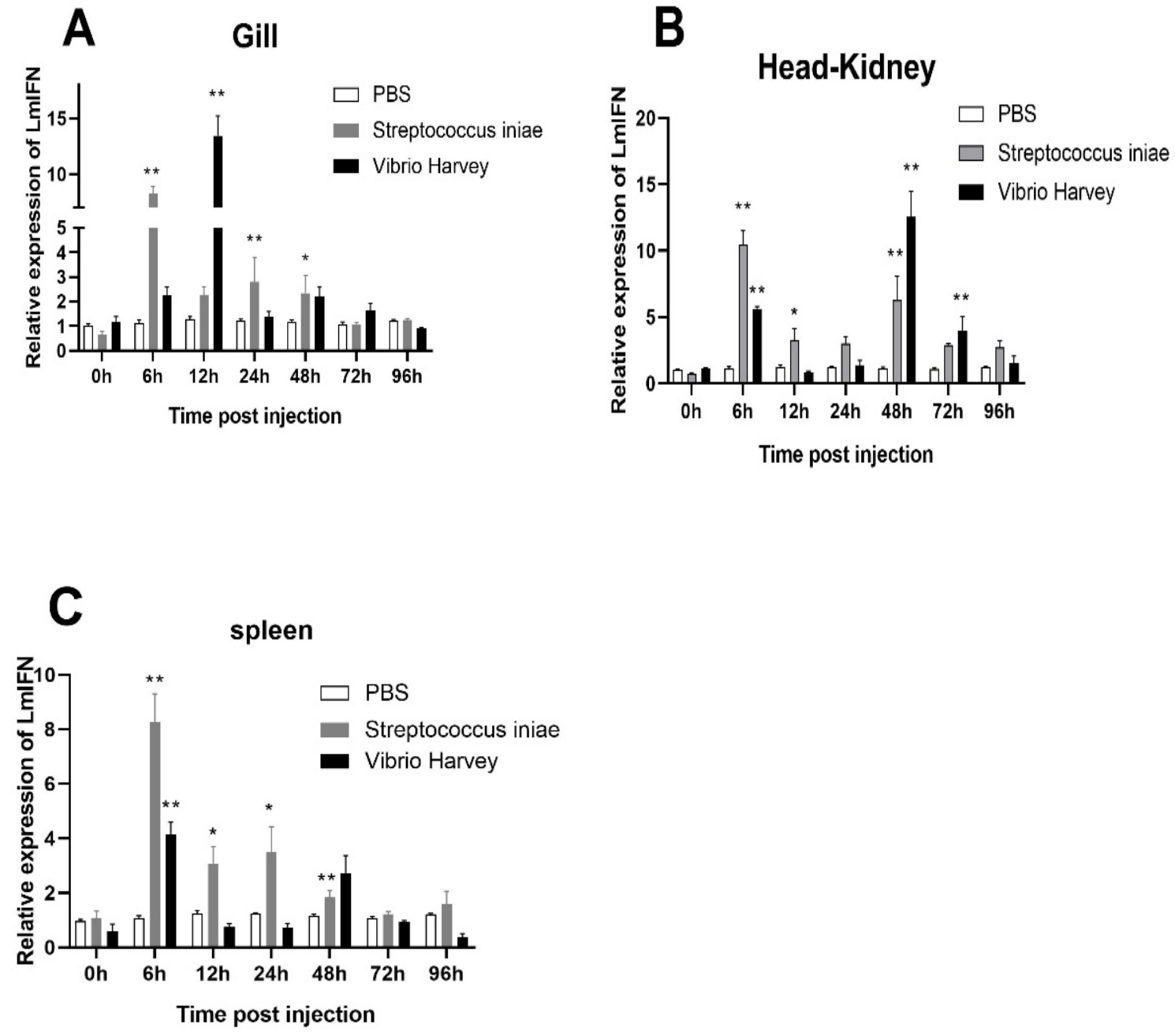

Figure 4 Spatiotemporal expression of LmIFNd mRNA in gill (A), head kidney (B) and spleen (C) of L. maculatus stimulated by Streptococcus iniae and Vibrio Harvey. The bars represent the mean \pm SD $(\mathrm{n}=3)$. * represents significant difference $(P<0.05)$, and $* *$ represents highly significant difference $(P<0.01)$.

\section{Discussion}

IFN is a kind of innate immune cytokine that is induced and plays an important role in various protective responses of the body to viral and microbial infection (Pereiro et al., 2014). Type I IFN is the main coordinating factor in the antiviral response of mammals and fish (Pereiro et al., 2014). In this study, the full-length LmIFNd cDNA sequence of $L$. maculatus was cloned using the RACE technique, which predicted to encode 185 amino acids with a molecular weight of $20.977 \mathrm{kDa}$ and a theoretical isoelectric point of 6.35 . The LmIFNd protein consists of a predictive signal peptide and a mature region, which is secreted into extracellular space through a classical protein secretion pathway, which is consistent with the IFN gene of most fish. However, there are type I IFN variants lacking a signal peptide in some fish (Mossman et al., 2013). LmIFNd has two cysteine residues and two glycosylation sites (Pereiro et al., 2014). Two Cys residues of LmIFNd may form disulfide bonds to stabilize the protein structure. Studies have shown that the function of ZfIFN lacking glycosylation sites in $D$. rerio still have antiviral activity, indicating that 
glycosylation sites in LmIFNd may not be related to antiviral function. But the specific functions of glycosylation sites in IFNs require further study (Altmann SM, 2003).

The results of tissue distribution showed that the highest relative expression was in the head-kidney, followed by the spleen and gill. A relatively low expression pattern was detected in the brain, skin, intestine, and heart. The results showed that LmIFNd was ubiquitously expressed in all tissues, although the expression levels varied. This means that LmIFNd may play a multifunctional role in different tissues of L. maculatus. In $S$. chuatsi, the expression of IFNc, IFNd and IFNh was highest in the head-kidney and lowest in the skin and muscle (Laghari et al., 2018), which was similar to the results of the current study. In turbot (Scophthalmus maximus), the expression of IFN1 was highest in the gill and lowest in the liver. The expression of IFN2 in was highest in the muscle and skin, but lowest in the head-kidney and liver (Pereiro et al., 2014). These results for $S$. maximus were significantly different from the results of the current study, possibly due to differences in immune function between species.

Type I IFN is a key molecule in the body's protective immune response against viruses, which controls the specific response of downstream genes and induces high levels of ISG expression, thus reducing virus proliferation (Platanias, 2005). Many studies have shown that IFN expression could be induced by viruses and Poly (I:C). For example, expression levels of Turbot IFN1 increased after viral infection, revealing a fold-change of about 150 at $72 \mathrm{~h}$ (Pereiro et al., 2014). It has been proven that the relative expression level of IFNa in the head kidney cells of sevenband grouper (Epinephelus septemfasciatus) stimulated by Poly(I:C) was significantly upregulated compared with a control group. Poly(I:C) could upregulate the expression of CiIFN mRNA in all detected tissues and peaked in the head kidney at $24 \mathrm{~h}$ after treatment, with expression levels 23.90 times higher than the control group ( $\mathrm{Li}$ et al., 2012). In the current study, it was found that the relative expression levels of LmIFNd were upregulated in the gill, head-kidney and spleen after injection with RGV and Poly (I:C). The fold-change was highest in the head kidney, similar to findings for $E$. septemfasciatus and $C$. idella, indicating that the head kidney plays an important role in antiviral immunity. This indicated that LmIFNd may be involved in defending RGV infection and plays an important role against virus infection in $L$. maculatus.

At present, the defense mechanism of type I IFNs against bacterial pathogens is still poorly understood. It has been shown that many bacteria can induce the upregulation of IFN expression, but there are species differences in their stimulating effects on the body. It has been reported that the expression level of type I IFN in fish increased significantly after lipopolysaccharide (LPS) or bacterial stimulation (Monroe et al., 2010). In D. rerio, injection of IFN recombinant protein can protect $D$. rerio from death caused by $S$. iniae stress (Lopez-Munoz et al., 2009). However, in S. maximus, when the bacterial suspension was administrated, a significant upregulation of IFN expression was recorded at $72 \mathrm{~h}$, but the administration of the plasmids encoding the $S$. maximus IFNs did not significantly affect the survival of individuals after a highly lethal dose of Aeromonas salmonicida. This result showed that there was no protective effect on the invasion of $A$. salmonicida (Pereiro et al., 2014). In this study, the gram-positive bacteria $V$. harvei and the gram-negative bacteria S. iniae were injected into L. maculatus. The expression of LmIFNd was upregulated in the immune tissues (gill, head-kidney and spleen) after stimulation of $V$. harveyi, indicating that LmIFNd may have a protective against bacterial infection.

\section{Acknowledgements}

We would like to thank Yukun Jie and Haoxiang Chen for their help with sampling of L. maculatus. We thank International Science Editing http://www.internationalscienceediting.com for editing this manuscript. This work was supported by the Central Public-interest Scientific Institution Basal Research Fund, CAFS (NO. 2020TD21, 2020XT09, and 2019ZD0501,), the Natural Science Foundation of Guangdong Province, China (NO. 2018A030313137), the Guangzhou Municipal Science and Technology Project (NO. 201906010028), and the National Natural Science Foundation of China, China (NO. 31802281). 


\section{References}

Altmann SM, M. M., Distel DL, et al. 2003. Molecular and functional analysis of an interferon gene from the zebrafish. Danio rerio. J Virol, 77, 1992-2002.

DOI: $10.1128 /$ jvi.77.3.1992-2002.2003.

Bae, H., Barlow, A. T., Young, H., \& Valencia, J. C. (2016). Interferon Y: an overview of its functions in health and disease. Encyclopedia of Immunobiology (pp. 494500). DOI: 10.1016/b978-0-12-374279-7.10006-2.

Casani, D., Randelli, E., Costantini, S., Facchiano, A. M., Zou, J., Martin, S., Secombes, C. J., Scapigliati, G., \& Buonocore, F. 2009. Molecular characterisation and structural analysis of an interferon homologue in sea bass (Dicentrarchus labrax L.). Mol Immunol, 46(5), 943-952.

DOI:10.1016/j.molimm.2008.09.016.

Chang, M., Nie, P., Collet, B., Secombes, C. J., \& Zou, J. 2009. Identification of an additional two-cysteine containing type I interferon in rainbow trout Oncorhynchus mykiss provides evidence of a major gene duplication event within this gene family in teleosts. Immunogenetics, 61(4), 315-325. DOI:10.1007/s00251-0090366-y.

Hou, Q., Gong, R., Liu, X., Mao, H., Xu, X., Liu, D., Dai, Z., Wang, H., Wang, B., \& Hu, C. 2017. Poly I:C facilitates the phosphorylation of Ctenopharyngodon idellus type I IFN receptor subunits and JAK kinase. Fish Shellfish Immunol, 60, 13-20. DOI: $10.1016 /$ j.fsi.2016.10.042.

Jin, Y., Zhou, T., Li, N., Liu, S., Xu, X., Pan, Y., Tan, S., Shi, H., Yang, Y., Yuan, Z., Wang, W., Luo, J., Gao, D., Dunham, R., \& Liu, Z. 2018. JAK and STAT members in channel catfish: identification, phylogenetic analysis and expression profiling after Edwardsiella ictaluri infection. Dev Comp Immunol, 81, 334-341. DOI:10.1016/j.dci.2017.12.019.

Laghari, Z. A., Chen, S. N., Li, L., Huang, B., Gan, Z., Zhou, Y., Huo, H. J., Hou, J., \& Nie, P. 2018. Functional, signalling and transcriptional differences of three distinct type I IFNs in a perciform fish, the mandarin fish Siniperca chuatsi. Dev Comp Immunol, 84, 94-108. DOI:10.1016/j.dci.2018.02.008.

Li, D., Lin, G., Yu, X., Huang, S., Lai, Q., Liu, Y., Wu, Z., \& Hu, C. 2013. Immunoprotection of grass carp (Ctenopharyngodon idella) with recombinant interferon (rCiIFN) against GCHV infection. Aquaculture, 388-391, 42-48.

DOI:10.1016/j.aquaculture.2013.01.017.

Li, D., Tan, W., Ma, M., Yu, X., Lai, Q., Wu, Z., Lin, G., \& Hu, C. 2012. Molecular characterization and transcription regulation analysis of type I IFN gene in grass carp (Ctenopharyngodon idella). Gene, 504(1), 31-40.

DOI:10.1016/j.gene.2012.04.091.

Li, L., Chen, S. N., Laghari, Z. A., Huang, B., Huo, H. J., Li, N., \& Nie, P. 2019. Receptor complex and signalling pathway of the two type II IFNs, IFN-Y and IFNyrel in mandarin fish or the so-called Chinese perch Siniperca chuatsi. Dev Comp Immunol, 97, 98-112. DOI:10.1016/j.dci.2019.03.016.

Long, S., Milev-Milovanovic, I., Wilson, M., Bengten, E., Clem, L. W., Miller, N. W., \& Chinchar, V. G. 2006. Identification and expression analysis of cDNAs encoding channel catfish type I interferons. Fish Shellfish Immunol, 21(1), 42-59. DOI: 10.1016/j.fsi.2005.10.008.

Lopez-Munoz, A., Roca, F. J., Meseguer, J., \& Mulero, V. 2009. New insights into the evolution of IFNs: zebrafish group II IFNs induce a rapid and transient expression of IFN-dependent genes and display powerful antiviral activities. J Immunol, 182(6), 3440-3449. DOI:10.4049/jimmunol.0802528.

Luo, K., Zhang, S., Tang, D., Xia, L., Gao, W., Tian, G., Qi, Z., Xu, Q., \& Zhang, w. 2018. Analysis of the expression patterns of the cytokine receptor family B (CRFB) and interferon gamma receptor (IFNGR) in Dabry's sturgeon (Acipenser dabryanus). Dev Comp Immunol, 84, 420-426. DOI:10.1016/j.dci.2018.03.011. 
Lutfalla, G., Crollius, H. R., \& Stange-thomann, N. 2003. Comparative genomic analysis reveals independent expansion of a lineage-specific gene family in vertebrates: The class II cytokine receptors and their ligands in mammals and fish. Bmc Genomics, 4(29). DOI:10.1186/1471-2164-4-29.

Milne, D. J., Campoverde, C., Andree, K. B., Chen, X., Zou, J., \& Secombes, C. J. 2018. The discovery and comparative expression analysis of three distinct type I interferons in the perciform fish, meagre (Argyrosomus regius). Dev Comp Immunol, 84, 123-132. DOI:10.1016/j.dci.2018.02.001.

Monroe, K. M., McWhirter, S. M., \& Vance, R. E. 2010. Induction of type I interferons by bacteria. Cell Microbiol, 12(7), 881-890. DOI:10.1111/j.1462-

5822.2010.01478.x

Mossman, K. L., Chang, M.-X., Zou, J., Nie, P., Huang, B., Yu, Z., Collet, B., \&

Secombes, C. J. 2013. Intracellular interferons in Fish: a unique means to combat viral infection. PLoS Pathogens, 9(11).

DOI: $10.1371 /$ journal.ppat.1003736.

Pereiro, P., Costa, M. M., Diaz-Rosales, P., Dios, S., Figueras, A., \& Novoa, B. 2014. The first characterization of two type I interferons in turbot (Scophthalmus maximus) reveals their differential role, expression pattern and gene induction. Dev Comp Immunol, 45(2), 233-244. DOI:10.1016/j.dci.2014.03.006.

Pestka S, K. C., Walter MR. 2004. Interferons, interferon-like cytokines, and their receptors. Immunol Rev, 202, 8-32. DOI:10.1111/j.0105-2896.2004.00204.X.

Platanias, L. C. 2005. Mechanisms of type-I-and type-II-interferon-mediated signalling. Nat Rev Immunol, 5(5), 375-386. DOI:10.1038/nri1604.

Purcell, M. K., Laing, K. J., Woodson, J. C., Thorgaard, G. H., \& Hansen, J. D. 2009. Characterization of the interferon genes in homozygous rainbow trout reveals two novel genes, alternate splicing and differential regulation of duplicated genes. Fish Shellfish Immunol, 26(2), 293-304. DOI:10.1016/j.fsi.2008.11.012.

Sadler, A. J., \& Williams, B. R. 2008. Interferon-inducible antiviral effectors. Nat Rev Immunol, 8(7), 559-568. DOI:10.1016/j.fsi.2008.11.012.

Schoenborn, J. R., \& Wilson, C. B. (2007). Regulation of interferon-y during innate and adaptive immune responses. Adv Immunol, 96, 41-101. DOI:10.1016/s0065-

2776(07)96002-2.

Secombes, C. J., \& Zou, J. 2017. Evolution of interferons and interferon receptors. Front Immunol, 8, 209. DOI:10.3389/fimmu.2017.00209

Stark GR, D. J. J. 2012. The JAK-STAT pathway at twenty. Immunity, 36, 503-514. DOI: $10.1016 /$ j.immuni.2012.03.013.

Stein, C., Caccamo, M., Laird, G., \& Leptin, M. 2007. Conservation and divergence of gene families encoding components of innate immune response systems in zebrafish. Genome Biol, 8(11). DOI:10.1186/gb-2007-8-11-r251.

Samuel, C. E. 2001. Antiviral actions of interferons. Clin Microbiol Rev, 14(4), 778-809, table of contents. DOI:10.1128/CMR.14.4.778-809.2001.

Taylor, G. A., Feng, C. G., \& Sher, A. 2004. P47 GTPases: regulators of immunity to intracellular pathogens. Nat Rev Immunol, 4(2), 100-109. DOI:10.1038/nri1270.

Teijaro, J. R. 2016. Type I interferons in viral control and immune regulation. Curr Opin Virol, 16, 31-40. DOI:10.1016/j.coviro.2016.01.001.

Zou, J., \& Secombes, C. J. 2011. Teleost fish interferons and their role in immunity. Dev Comp Immunol, 35(12), 1376-1387. DOI:10.1016/j.dci.2011.07.001. 